\title{
TOPOLOGY OF STEADY HEAT CONDUCTION IN A SOLID SLAB SUBJECT TO A NONUNIFORM BOUNDARY CONDITION: THE CARSLAW-JAEGER SOLUTION REVISITED
}

\author{
R. G. KASIMOVA ${ }^{\otimes 1}$ and YU. V. OBNOSOV ${ }^{2}$ \\ (Received 7 June, 2012; revised 19 October, 2012; first published online 18 February, 2013)
}

\begin{abstract}
Temperature distributions recorded by thermocouples in a solid body (slab) subject to surface heating are used in a mathematical model of two-dimensional heat conduction. The corresponding Dirichlet problem for a holomorphic function (complex potential), involving temperature and a heat stream function, is solved in a strip. The Zhukovskii function is reconstructed through singular integrals, involving an auxiliary complex variable. The complex potential is mapped onto an auxiliary half-plane. The flow net (orthogonal isotherms and heat lines) of heat conduction is compared with the known Carslaw-Jaeger solution and shows a puzzling topology of three regimes of energy fluxes for temperature boundary conditions common in passive thermal insulation. The simplest regime is realized if cooling of a shaded zone is mild and heat flows in a slightly distorted "resistor model" flow tube. The second regime emerges when cooling is stronger and two disconnected separatrices demarcate the back-flow of heat from a relatively hot segment of the slab surface to the atmosphere through relatively cold parts of this surface. The third topological regime is characterized by a single separatrix with a critical point inside the slab, where the thermal gradient is nil. In this regime the back-suction of heat into the atmosphere is most intensive. The closed-form solutions obtained can be used in assessment of efficiency of thermal protection of buildings.
\end{abstract}

2010 Mathematics subject classification: primary 35J25; secondary 65E04, 80A05.

Keywords and phrases: two-dimensional heat conduction, Laplace's equation, heat lines, isotherms, complex potential, conformal mappings.

\section{Introduction}

Analytical solutions for heat conduction in solid bodies are needed in different engineering designs involving heat transfer [3]. Steady conduction can be quantified

\footnotetext{
${ }^{1}$ German University of Technology in Oman, Muscat, Sultanate of Oman;

e-mail: rouzalia.kasimova@gutech.edu.om.

${ }^{2}$ Institute of Mathematics and Mechanics, Kazan Federal University, Kazan, Russia;

e-mail: yurii.obnosov@gmail.com.

(C) Australian Mathematical Society 2013, Serial-fee code 1446-1811/2013 \$16.00
} 
and visualized by a flow net composed of isotherms and heat lines, proposed by Bejan [2] for general convective heat transfer regimes. In two-dimensional conduction, the two families of curves constituting the net are orthogonal because the temperature field is governed by the Laplace equation, similar to corresponding nets in dynamics of ideal fluids, electrostatics and magnetostatics, diffusion of tracers and Darcian porous media flows.

Analytical solutions to potential field problems, where the intricate topology of $2 \mathrm{D}$ flow nets is controlled by internal heterogeneities of the domain, but the boundary conditions are uniform or follow from the symmetry principle in cases of internal heat sources/sinks, have recently been obtained [6, 9-12]. In these cases, an elementary cell, where the potential problems were solved, represents a flow tube, which consists of two isotherms and two adiabatic lines. Inside the flow tube, streamlines and isotherms can be drawn by hand, and experienced engineers used to do so in the pre-computer epoch with good accuracy. In practical engineering, especially in construction engineering, a solid body (wall, roof, parapet, etc.) exposed to a heat load is often considered homogeneous with respect to thermal conductivity, but the boundary conditions are nonuniform; that is, inherently nonisothermic and nonadiabatic surfaces confine the conduction domain. (This is caused, for instance, by different exposure of solid body surfaces to solar radiation.) Then reconstruction of the topology of heat flow is not so simple as in flow tubes (and sometimes gives even counterintuitive results). Therefore, a detailed analysis of the field and computer-aided visualization of the net is mandatory.

In this paper we study the effect of a variable-temperature boundary condition imposed on the surface of a very simple geometrical figure: a slab. We implement the theory of boundary-value problems for holomorphic functions [4] and arrive at an explicit, closed-form solution for the complex potential (temperature and stream function), which refurbishes the known solution in terms of temperature, obtained by Carslaw and Jaeger [3].

A practical motivation for our study stems from the passive thermal insulation of building roofs in the hot climates of Oman and Australia. We consider a roof whose upper surface is exposed to direct solar radiation as shown in Figure 1 (vertical section of the slab) and whose internal surface (building interior) is air-conditioned and kept at a relatively low temperature. In order to reduce the heat load (and monthly utility bills), shading by a thermal barrier, which covers a part of the external slab surface, is used. In our model, we average the diurnal temperature swings, recorded by thermocouples on the surface of a concrete slab, and study the corresponding steady (average) 2D temperature distribution and heat flux in the slab, which, due to partial shading, are induced by a nonconstant boundary condition for temperature.

\section{Mathematical model and analytical solution}

We consider a vertical cross-section of the slab of thickness $b$ and thermal conductivity $k$, and a thermal barrier $\mathrm{E}_{1} \mathrm{OE}_{2}$ (in practice, strip-type shading against 


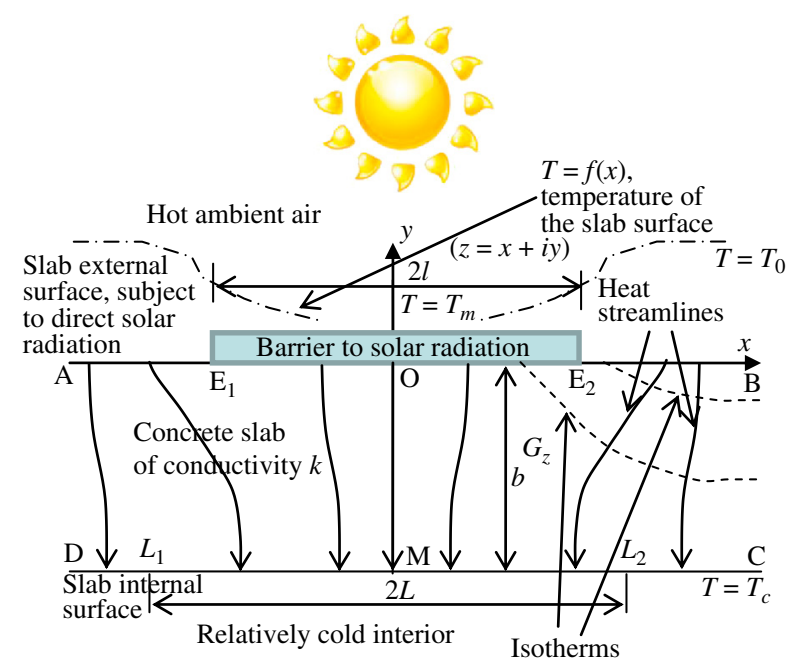

FIGURE 1. Vertical cross-section of a slab with a thermal barrier.

solar radiation ${ }^{1}$ ). Figure 1 shows a vertical cross-section and Cartesian coordinates. Far from the barrier on the rays $\mathrm{AE}_{1}$ and $\mathrm{E}_{2} \mathrm{~B}$, the slab temperature $T_{0}$ is constant (daily average).

Along AOB, we have experimental data (temperature) obtained by thermocouples and we take the daily averages of these values. The $x$-distribution of this average temperature is a single-minimum function $f(x)$. This function is symmetric $(f(-x)=$ $f(x)$ ) for common shading and $f(x) \rightarrow T_{0}$ as $x \rightarrow \pm \infty$. (These asymptotics are also confirmed in experiments with shading by cardboard boxes and other lightweight objects; see Figure 2.)

We define $F(x)$ by

$$
f(x)=T_{0}-F(x) \quad \text { at } y=0,
$$

where $F(x)$ is a single-maximum $\left(T_{M}=T_{0}-T_{m}\right)$ function shown in Figure 3(a). We assume that along the internal surface (DC in Figure 1) temperature is constant, $T_{c}$ :

$$
T=T_{c} \quad \text { at } y=-b .
$$

In the case of no thermal barrier in Figure 1, heat conduction in the slab of Figure 1 is trivially 1D. With the barrier, the so-called "thermal resistor" models (see Sailor et al. [15]) have been used. The resistor approximation assumes the AOB boundary condition to be a step-function (reflecting the barrier width) and the heat streamlines

\footnotetext{
${ }^{1}$ One may ask: why not shade the whole roof surface? The answer is: (a) full shading is more costly; (b) full shading is heavier and in retrofitted buildings there is a limitation imposed by structural stability (weight of extra load); (c) roofs in Oman host an air-conditioning unit, water supply tank, satellite dishes, etc., which need access potentially obstructed by shading elements.
} 
(a)

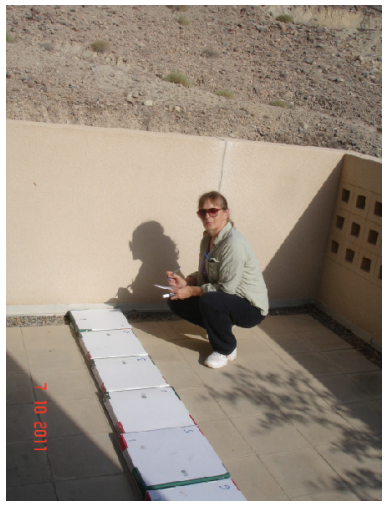

(b)

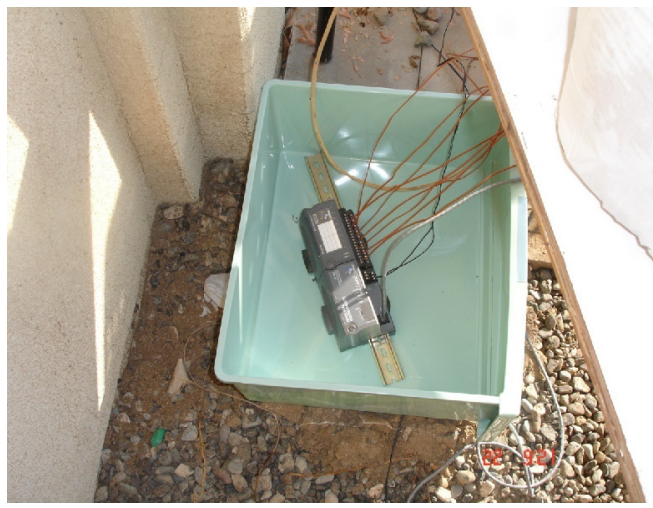

Figure 2. (a) Experimental strip of cardboard boxes for shading. (b) Data logger with eight thermocouple wires for retrieving temperature readings from beneath the boxes and nonshaded roof area.

are postulated to be straight and perpendicular to both slab boundaries, that is, heat flow is again 1D. Our objective is to assess analytically the spatial nonuniformity of temperature and heat lines (within the slab) caused by the experimental boundary condition (2.1).

According to the Fourier law, heat conduction in the strip AOBCMD (denoted $G_{z}$ ) of Figure 1 is governed by

$$
\vec{J}=-k \nabla T(x, y)
$$

where $\vec{J}(x, y)$ is the heat flux vector with a vertical component $v$ and horizontal component $u$.

We introduce a complex physical coordinate $z=x+i y$ and a complex potential $w=\phi+i \psi$, where $i$ is an imaginary unit, $\phi=-k\left(T-T_{c}\right)$ is the potential and $\psi$ is a stream function, which is related to $\phi$ through the Cauchy-Riemann conditions:

$$
\frac{\partial \phi}{\partial x}=\frac{\partial \psi}{\partial y}=u, \quad \frac{\partial \phi}{\partial y}=-\frac{\partial \psi}{\partial x}=v .
$$

The heat lines with $\psi$ constant allow a better visualization of heat transfer and an assessment of thermophysical efficiency [2]. Both $\phi$ and $\psi$ are harmonic,

$$
\Delta \phi(x, y)=0, \quad \Delta \psi(x, y)=0,
$$

and $w(z)$ is a holomorphic function.

An integral solution of the boundary-value problem (2.1), (2.2), and (2.4) is given by Carslaw and Jaeger [3] (Chapter V, Section 3, equation 2.19). Here we derive an alternative solution. Carslaw and Jaeger [3] obtained their solution by the Fourier transform method. The Laplace equation is commonly solved in a stream tube $G_{z}$ by separation of variables and Fourier series expansions [3, 5]. The Fourier method is, however, limited to the domains $G_{z}$ consisting of two constant temperatures: two 
(a)

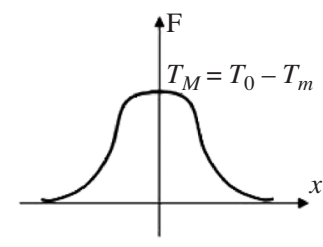

(b) $\quad(w=\phi+i \psi)$

(c)

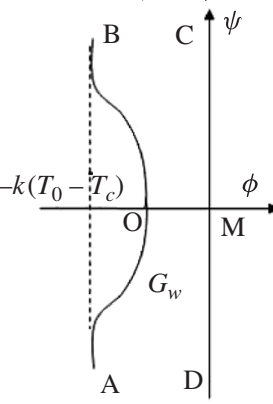

FIGURE 3. (a) Temperature boundary condition on the exterior surface (the density of the Cauchy integral); (b) complex potential domain for small $T_{M}$; (c) auxiliary plane where the Dirichlet problem is solved.

adiabatic segments ("rectangles"), as boundaries coinciding with the level lines of a Cartesian, cylindrical, spherical, etc. coordinate system, where the Laplace equation separates. In other words, Carslaw and Jaeger in their book used Fourier series and integrals to solve the ODEs to which Laplace's equation is reduced in a rectangle (in particular, strip or half-strip). Our method does not have this "rectangle" limitation and is applicable to any $G_{z}$-polygon with arbitrary mixed (Dirichlet-Neumann) boundary conditions.

Without loss of generality we assume that $\psi=0$ along OM, which follows from the symmetry of $f(x)$. The isotherms (equipotential lines, $\phi$ constant) are dashed in Figure 1 and heat streamlines ( $\psi$ constant) are shown with arrows indicating the direction of heat transfer. The domain $G_{z}$ is obviously fixed but the domain corresponding to $G_{z}$ in the $w$-plane, $G_{w}$, depends on $f(x)$ and is surprisingly complex even for simple heating regimes, that is, functions $f(x)$.

If $T_{m}$ is close to $T_{0}$ and the slope of $f(x)$ is small, then $G_{w}$ is a strip with a slightly bulging side AOB (Figure 3(b)). The streamlines in $G_{z}$ (Figure 1) are somewhat curved, mostly in the slab zones where the imposed $f(x)$ has a relatively high magnitude of slope, $|d f / d x|$ (see Figure 1). This regime is topologically close to what the "resistor" model [15] approximates.

For a smaller $T_{m}$ (fixed $T_{0}$ but higher $T_{M}$ ) and/or stronger variation of the slope of $f(x)$, the topology of heat lines is shown in Figure 4(a). On AOB (we recall that $f(x)$ is a single-minimum function) at four points $\mathrm{H}_{1}, \mathrm{H}_{2}, \mathrm{H}_{3}$, and $\mathrm{H}_{4}$, the direction of the $v$-component of the thermal gradient changes from inside the slab to the exterior. Indeed, along $\mathrm{AS}_{2} \mathrm{H}_{1}$ and $\mathrm{BS}_{3} \mathrm{H}_{4}$ heat is conducted from the exterior surface into the slab. Along $\mathrm{H}_{1} \mathrm{H}_{2}$ and $\mathrm{H}_{4} \mathrm{H}_{3}$ heat is discharged back and along $\mathrm{H}_{2} \mathrm{H}_{3}$ heat moves from the exterior surface to the interior. There are two separatrices (dividing streamlines shown as thick lines) $\mathrm{S}_{2} \mathrm{H}_{2} \mathrm{E}_{2}$ and $\mathrm{S}_{3} \mathrm{H}_{3} \mathrm{E}_{3}$, which demarcate five different zones in $G_{z}$. The corresponding domain $G_{w}$ is shown in Figure 4(b) where the image of AOB is a 
(a)

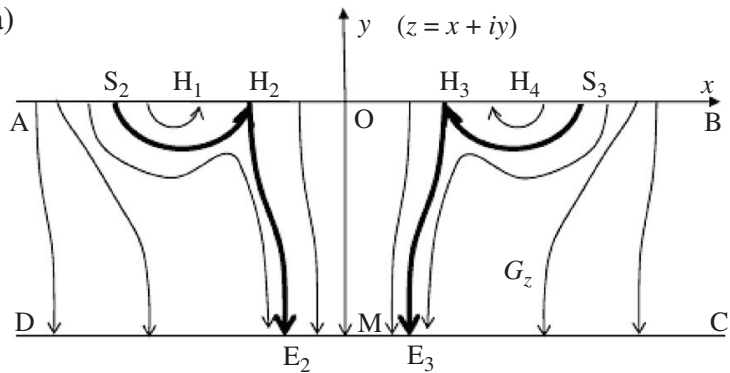

(b)

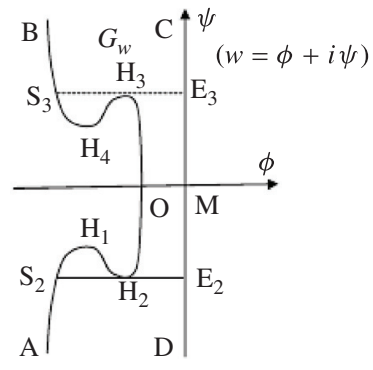

FigurE 4. (a) Heat line topology with four hinge points, and (b) the corresponding knob-shaped bounded complex potential domain.

(a)

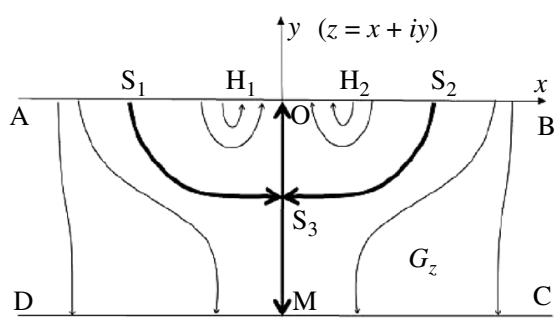

(b) $\quad(w=\phi+i \psi)$

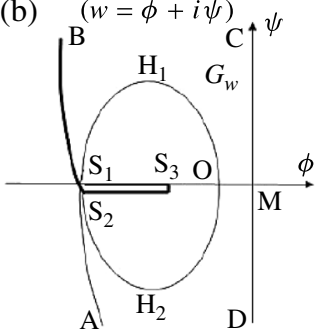

Figure 5. (a) Heat line topology with two hinge points, and (b) the corresponding double-sheet Riemann surface as the complex potential domain.

knob-shaped curve. So, in the regime of Figure 4, heat from the hot, unprotected slab surface is partially "sucked" back to the ambient air owing to the barrier in Figure 1.

For even smaller $T_{m}$ and/or stronger slopes of $f(x)$ we arrive at the topology depicted in Figure 5(a). Here we have two points $\mathrm{H}_{1}$ and $\mathrm{H}_{2}$ where the flow changes its orientation from the interior to the exterior of the slab. The only separatrix (shown as thick lines in Figure 5(a)) has a saddle point $S_{3}$. Above $S_{1} S_{3} S_{2}$ heat is circulated from the air into the concrete and back, without entering the building interior. The domain $G_{w}$ shown in Figure 5(b) is a double-sheet Riemann surface. The second sheet $\mathrm{S}_{1} \mathrm{H}_{1} \mathrm{OH}_{2} \mathrm{~S}_{2}$ is stitched to the first (main) sheet through the cut $\mathrm{S}_{1} \mathrm{~S}_{3} \mathrm{~S}_{2}$, which images the separatrix in $G_{z}$. In Figure 5(b) we purposely distorted the branch $\mathrm{AS}_{1}$ (of course, this branch in $G_{w}$ is symmetrical to $\mathrm{S}_{2} \mathrm{~B}$ with respect to the $\phi$ axes) in order to illustrate the stitching of the second sheet. Points $S_{1}$ and $S_{2}$ are located on the opposite sides of the cut in $G_{w}$.

The regime of Figure 5 is most favourable for thermal insulation of the building because the "back-suction" of the heat under the barrier is the strongest. However, here a dilemma emerges: the temperature of the interior $\left(T_{c}\right)$ is almost beyond engineering control because it is determined by standards of comfort for human beings (about $22^{\circ} \mathrm{C}$ ). In practice, an engineer can reduce only $T_{m}$ (Figure 1 ) in order to redirect the heat flux back as in Figures $4-5$. Shading reduces $T_{m}$ but has a limit and, if not smartly 
designed, can have a negative impact on the nocturnal back-radiation. Semi-passive thermal insulation (for example, by using latent heat flux derived from evaporation: water spraying over certain roof zones) can reduce $T_{m}$ more than simple shading, but then the running cost of sprayed water in hot Omani conditions can be prohibitively expensive. Mathematically, $T_{m}$ can be made arbitrarily small but physically installing a "freezing" device on the roof is not feasible.

If $T_{M}<T_{c}<T_{0}$, then still another heat conduction regime is obtained, with heat flux from the interior. This regime may occur in cold countries, like Germany, when the building interior is heated and the ambient air is cold; this has not been experimentally observed in Oman where, for example, in Muscat the annual average air temperature is $31^{\circ} \mathrm{C}$ and mean (annual) total heat flux exceeds $600 \mathrm{~mW} / \mathrm{cm}^{2}$. Consequently, this regime is not discussed here. Similarly warm and sunny climatic environments are common in several regions of Central and Western Australia (see the Australian Bureau of Meteorology website [1]).

We now implement a mathematical technique which can readily tackle the regime in Figures 3-5. The method is based on: (1) a conformal mapping of one domain $\left(G_{z}\right.$ in our case) onto an auxiliary domain (circle, half-plane); and (2) solving there a Dirichlet, mixed, Newton (Robin) or refraction problem (with the first to fourth boundary conditions, respectively) and further reconstruction of the second holomorphic function in the auxiliary domain.

So, first, we map $G_{z}$ conformally onto the upper half-plane $\operatorname{Im} \varsigma>0$ of an auxiliary plane $\varsigma=\xi+i \eta$ shown in Figure 3(c) by the elementary function

$$
z=-\frac{b}{\pi} \log \frac{1+\varsigma}{1-\varsigma}
$$

In this plane images of points $A$ and $D$ as well as $C$ and $B$ coincide (Figure 3(c)).

Next, we introduce the Zhukovskii function as [14]

$$
Z h=w-i\left(T_{0}-T_{c}\right) k z / b+k\left(T_{0}-T_{c}\right)=R+i I .
$$

The real and imaginary parts of this function are $R=\operatorname{Re} Z h=\phi+k\left(T_{0}-T_{c}\right) y / b+$ $k\left(T_{0}-T_{c}\right)$ and $I=\operatorname{Im} Z h=\psi-k\left(T_{0}-T_{c}\right) x / b$ respectively. Obviously, $Z h(z)$ is also holomorphic in the half-plane $\operatorname{Im} \xi>0$. The following boundary conditions, in accordance with (2.1), (2.2), hold for $Z h(z)$ on the real axis:

$$
R=0 \quad \text { for }|\xi|>1, \quad R=k F[x(\xi)] \text { for }|\xi|<1,
$$

where equation (2.5) gives $x(\xi)$ as

$$
x=-\frac{b}{\pi} \log \frac{1+\xi}{1-\xi}, \quad|\xi|<1 ; \quad x=-\frac{b}{\pi} \log \frac{\xi+1}{\xi-1}, \quad \xi>1 .
$$

Obviously (see Figure 3(a)), $F(\xi) \rightarrow 0$ as $\xi \rightarrow \pm 1$. The function $F(\xi)$ is interpolated from experimental (thermocouple) point-wise collected values averaged daily. 
We used $F(x)=T_{M} \exp \left[-a x^{2}\right]$, where $a$ is a fitting parameter, as an approximation for experimentally measured temperature values. Any other function, for example, $F(x)=T_{M} /\left(1+\left(b_{c} x\right)^{2}\right)$ (where $b_{c}$ is another fitting parameter), can be used in (2.6) as a boundary condition. The formula (2.7), obtained from the conformal mapping, is fixed and does not depend on interpolation of experimental data and the choice of $F(x)$.

The real part of the Zhukovskii function $R(\varsigma)$ satisfies the boundary conditions (2.6). At the point $M(\zeta \rightarrow \infty)$ the imaginary part of this complex function $I(\varsigma)=0$. Then an integral solution to the stated Dirichlet boundary-value problem $(2.6)$ is $[4,14]$ :

$$
Z h(\zeta)=\frac{1}{\pi i} \int_{-1}^{1} \frac{k F(\tau) d \tau}{\tau-\zeta}
$$

Passing to the Sokhotsky-Plemelj limit $\zeta \rightarrow \xi,-1<\xi<1$ from equation (2.8), we obtain the stream function along AOB:

$$
\psi=\frac{k\left(T_{0}-T_{c}\right)}{b} x(\xi)-\frac{1}{\pi} \int_{-1}^{1} \frac{k F(\tau) d \tau}{\tau-\xi} .
$$

Now it is clear that the purpose of introducing the Zhukovskii function as a linear combination of $z$ and $w$ was to obtain the Dirichlet problem of the simplest possible type (the reconstructed $Z h$ function vanishes at infinity while $w$ and $z$ do not).

We note that the integral in equation (2.9) is singular in the interval $-1<\xi<1$ (which corresponds to the line AOB in Figure 1) and should be calculated in the sense of principal value. Wolfram's Mathematica [16] has a corresponding routine CauchyPrincipalValue, which we used in numerical integration. At $|\xi|>1$ (line DMC in Figure 1) the integral in equation (2.9) is regular and we used the routine NIntegrate [16].

It is convenient to expand the kernel in equation (2.9) in a series of Chebyshev's polynomials [8] of the second kind:

$$
F(\tau)=T_{M} \sum_{n=1}^{\infty} b_{n} U_{n}(\tau), \quad|\tau|<1, \quad b_{n}=\frac{2}{\pi} \int_{-1}^{1} \frac{f(\tau) U_{n}(\tau)}{\sqrt{1-\tau^{2}}} d \tau,
$$

where $U_{n}(\tau)=\sin [n \arccos \tau]$. For any smooth function $F(\tau)$ (even belonging to the Hölder class is sufficient), this series is uniformly convergent on the interval $(-1,1)$. Then for the roof surface $\mathrm{AB}$, equation (2.9) is reduced to

$$
\psi=\frac{k\left(T_{0}-T_{c}\right)}{b} x(\xi)-k T_{M} \sum_{n=1}^{\infty} b_{n} T_{n}(\xi), \quad|\xi|<1,
$$

and for the ray MD,

$$
\psi=\frac{k\left(T_{0}-T_{c}\right)}{b} x(\xi)+k T_{M} \sum_{n=1}^{\infty} b_{n}\left(\xi-\sqrt{\xi^{2}-1}\right)^{n}, \quad \xi>1,
$$


where $T_{n}(\tau)=\cos [n \arccos \tau]$ are the Chebyshev polynomials of the first kind. In (2.11) the branch of the function $g(\zeta)=\zeta-\sqrt{\zeta^{2}-1}$, positive at $\xi>1$, is fixed in the plane with the cut along the segment $[-1,1]$. The fixed branch satisfies the identity $g(\zeta) \equiv g(-\zeta)$ for all $\zeta$ and in particular for $\zeta=\xi<-1$. Hence, for the ray MC $(\xi<-1)$ we have $\psi(-\xi)=\psi(\xi)$, that is, the function (2.11) can be used.

The vertical component of the thermal gradient is

$$
v=-\frac{\partial \psi}{\partial x}=-\frac{\partial \psi}{\partial \xi}\left(\frac{\partial x}{\partial \xi}\right)^{-1}
$$

Far from the insulation zone (large values of $|x|$ ) the horizontal component $u$ of the gradient vanishes and $v \rightarrow v_{\infty}=-k\left(T_{0}-T_{c}\right) / b$. We introduce a dimensionless vertical component $v^{*}=v / v_{\infty}$. On AOB, differentiation of (2.7) and (2.10) yields

$$
v^{*}(\xi)=1-\frac{\pi r}{2} \sqrt{1-\xi^{2}} \sum_{n=1}^{\infty} n b_{n} U_{n}(\xi), \quad|\xi|<1, r=\frac{T_{M}}{T_{0}-T_{c}} .
$$

Then the hinge points (if they exist for a given $f(x)$ ) in Figures 2(a) and 3(a) are found from (2.12) as the roots of the equation $v^{*}(\xi)=0,|\xi|<1$. We solved this equation using the FindRoot routine of Mathematica [16].

How much in terms of total energy saving do we gain from thermal insulation? In order to answer this question we select two symmetrical points $L_{1}$ and $L_{2}$ on DC (Figure 1), distance $2 L$ apart. Without the barrier in Figure 1 (1D conduction), the total heat entering the interior (per unit length in the direction perpendicular to the plane in Figure 1) through a strip of width $2 L$ is $Q_{0}=2 L k\left(T_{0}-T_{c}\right) / b$. From the definition of the stream function the total heat flowing through the same area but in $2 \mathrm{D}$ conduction with insulation is $Q=2 \psi_{L 1}$, where $\psi_{L 1}$ is directly expressed from (2.7) and (2.11) as

$$
\psi_{L 1}=\frac{k\left(T_{c}-T_{0}\right) L}{b}+k T_{M} \sum_{n=1}^{\infty} b_{n} \tanh ^{n}\left(\frac{\pi L}{4 b}\right) .
$$

We introduce a dimensionless energy saving through $\mathrm{L}_{1} \mathrm{~L}_{2}$ as $S(L)=\delta Q / Q_{0}$, where $\delta Q=Q_{0}+2 a \psi_{L 1}$ and, with $\psi_{L 1}$ taken from equation (2.13),

$$
\delta Q=r \frac{b}{L} \sum_{n=1}^{\infty} b_{n} \tanh ^{n}\left(\frac{\pi L}{4 b}\right)
$$

As we have pointed out, the $F(x)$ selected is $T_{M} \exp \left[-a x^{2}\right]$. Figure 6 shows $S$ as a function of a dimensionless width $L^{d}=L / b$ for $r=0.25$ and $a^{d}=0.025,0.1$ and 0.4 (curves $1-3$, respectively, where $a^{d}=a b^{2}$ ), calculated by equation (2.14). In Figure 7, $v^{d}$ is shown as a function of dimensionless abscissa $x^{d}=x / b$ along AOD for $r=0.5$ and $a^{d}=1,2$ and 4 (curves 1-3, respectively), calculated by (2.12). As we can see from Figure 7, for the $F(x)$ selected we have the flow topology of Figure 1 (no hinge points) for the first two curves and the two-hinge-points regime for the third curve. All three 


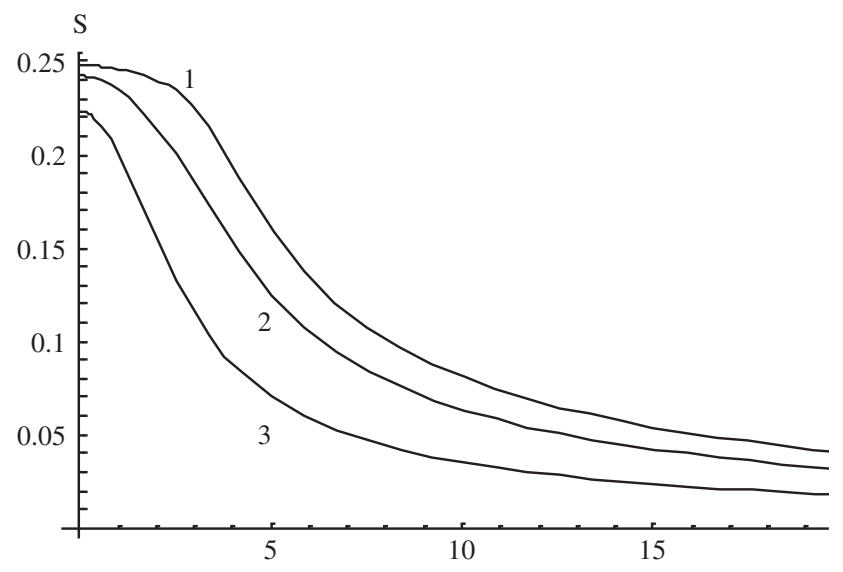

FIGURE 6. Energy saving factor $S$ as a function of $L^{d}$ for $r=0.25$ and $a^{d}=0.025,0.1$ and 0.4 (curves $1-3$, respectively).

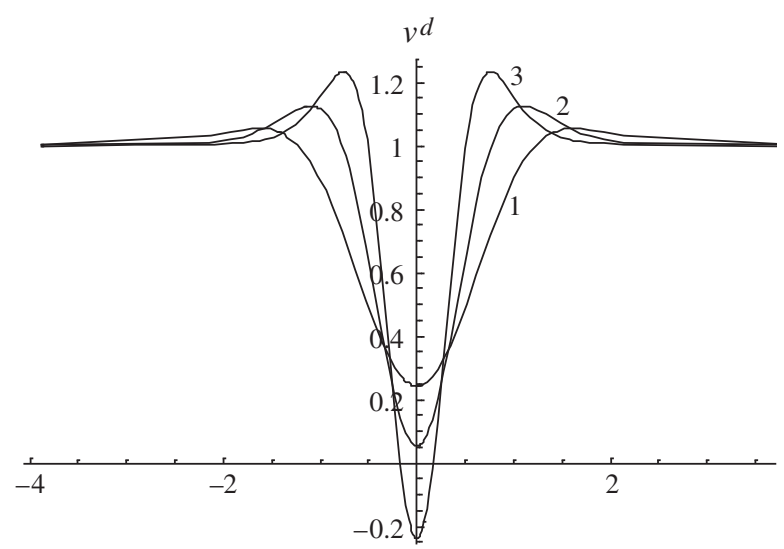

FIGURE 7. Vertical component of thermal gradient $v^{d}$ as a function of $x^{d}$ along AOD for $r=0.5$ and $a^{d}=1$, 2 and 4 (curves $1-3$, respectively).

curves have two blips (maxima) which indicate that in the near-blip zone of the roof the intensity of conduction into the slab is even higher than in the case of no thermal insulation, that is, near the edges $\mathrm{E}_{1}$ and $\mathrm{E}_{2}$ in Figure 1 the barrier "sucks" energy.

Without any series expansions we can use equations (2.5) and (2.8) directly in the following form:

$$
w^{d}=i z^{d}-1-\frac{i r}{\pi} \int_{-1}^{1} \frac{F(\tau) d \tau}{\tau+\tanh \left(\pi z^{d} / 2\right)},
$$

where dimensionless complex variables are introduced as $w^{d}=w /\left(k\left(T_{0}-T_{c}\right)\right), z^{d}=$ $z / b$. 


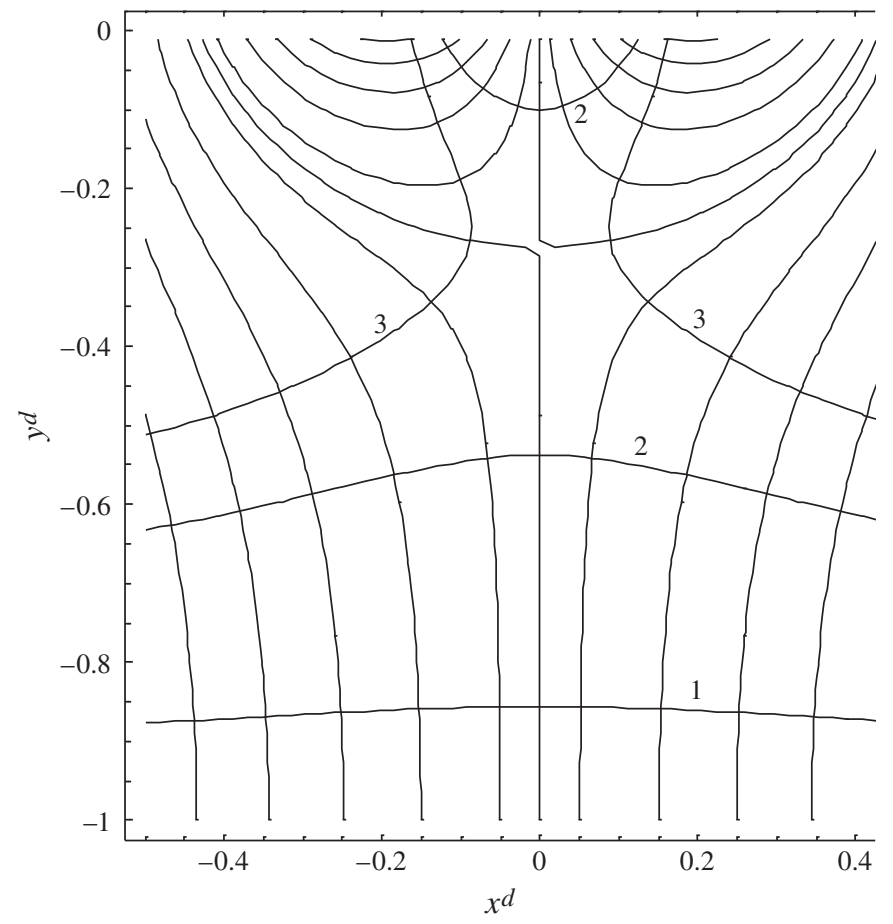

FIGURE 8. Flow net (isotherms and heat lines) for $F=\exp \left[-a^{d}\left(x^{d}\right)^{2}\right], r=0.9, a^{d}=15$.

With the help of the routines $\mathbf{R e}$ and Im [16] we separated the real and imaginary parts in equation (2.15). Then we used ContourPlot [16] to plot the flow nets. Figure 8 shows the flow net for $F=\exp \left[-a^{d}\left(x^{d}\right)^{2}\right]$ with $r=0.9$ and $a^{d}=15$ (two-hinge-points regime of Figure 5(a)). In Figure 8, in order to avoid clutter, only three equipotential contours are presented: $\phi^{d}=-0.1$ (curve 1, single branch, see the Riemann surface in Figure 5(b)), $\phi^{d}=-0.3$ (two branches, labeled 2) and $\phi^{d}=-0.4$ (two branches, labeled 3). For the sake of comparison we also plotted the equipotentials according to the Carslaw-Jaeger solution [3] mentioned earlier, which in our notation and dimensionless variables reads:

$$
\phi^{d}\left(x^{d}, y^{d}\right)=\frac{1}{2} \sin \left(\pi y^{d}\right) \int_{-\infty}^{\infty} \frac{1-r \exp \left[-a^{d} \tau^{2}\right]}{\cosh \left[\pi\left(1-y^{d}\right)\right]+\cosh \left[\pi\left(x^{d}-\tau\right)\right]} d \tau .
$$

Our equation (2.15) and equation (CJ-2.19), when plotted by Mathematica, give identical contours.

It is clear that $S_{3}$ in Figure 5(a) is indeed a saddle point, that is, if we approach this point from the left and right, then temperature decreases towards this point, but if we move from $\mathrm{S}_{3}$ upward and downward, then temperature increases. Mathematica 
contour-plotting computations confirmed what we conceptualized as flow topologies in Figures 1(a), 4(a), and 5(a).

Obviously, the complex topology of heat lines and isotherms is a great warning to practical engineers. Suppose, for instance, that one inserts two thermal probes in the interior of the slab and the tips of the probes strike the upper and lower branch of the isotherm 2 in Figure 8. Then, the interpretation of this reading, based on the inculcated "resistor" model of 1D heat flow will be totally misleading: the 1D model with such experimental data predicts no heat flow while in reality conduction is very intensive. Similar erroneous conclusions can be drawn from simplistic models used in inverse problems, for example for determination of thermal properties of the body (conductivity) if the heat flux is given and thermometry is used in a posited but physically inadequate 1D mathematical model.

\section{Conclusions}

Our solution, equation (2.15), gives the temperature and heat flux field in the slab as an output of the ContourPlot routine of a standard computer algebra package (Mathematica). The solution is simple, versatile and provides analytical expressions for isotherms, heat lines, and thermal gradient (magnitudes and directions). Our solution gives the same results as the known solution from Carslaw and Jaeger [3] (obtained by a different method, the flow net of which we easily reproduced by the built-in functions of Mathematica). The flow topology in Figures 4 and 5 is indeed counterintuitive and, to the best of our knowledge, has never been reported before. Indeed, in standard 1D approximations of flow tubes, heat is conducted from one (hot) boundary to another (cold) which precludes streamlines making Uturns and discharging heat back (into the exterior of a solid body) through a segment geometrically adjacent to one through which heat enters the body. Our approach to solving the corresponding boundary-value problem of heat conduction can be easily extended to more complex geometries of conducting elements, for example a rectangle or other polygons such as $G_{z}$, which can be mapped onto an auxiliary plane by the Schwartz-Christoffel formula. Another interesting question is on the pattern of shading, which results in minimization of the total heat transfer from the roof to the ceiling. In Figure 1 only $L$ is under our control, that is, we can make the barrier either broader or narrower. The temperature along $\mathrm{E}_{1} \mathrm{E}_{2}$ in Figure 1 and in the vicinity of the barrier then follows from experiments. One can, however, select periodic barriers. Then the heat conduction problem will be similar to one studied by Manners [7].

What are the weak points of our analysis? We neglected the transient effects of conduction, that is, we did not involve the diffusion (parabolic) equation. We tackled daily-average temperature values. Philip [13] has shown that with the period averaging, which we did, nontrivial phenomena (like overshooting) emerge only in nonlinear situations. Our $k$ does not depend on $T$, that is, the basic heat-transfer equation (2.3) leads to a linear (diffusion or Laplace's) PDE. Therefore, the field values of average temperature, which we have obtained by solving Laplace's equation, can 
be bounded from above and below by those representing the "extreme" values of the boundary condition (we recall that the Laplace and linear diffusion equations obey the maximum principle). In other words, resonances in the full transient model or Philip-type "superelevation" phenomena in the daily-averaged temperature field are not possible.

\section{Acknowledgements}

This work was supported by the German University of Technology in Oman and the Russian Foundation for Basic Research grant no. 12-01-97015-r_povolgh'e_a. Helpful comments by two anonymous referees are appreciated.

\section{References}

[1] Australian Government Bureau of Meteorology, at: http://www.bom.gov.au/index.shtml.

[2] A. Bejan, Convection heat transfer, 3rd edn. (Wiley, Hoboken, NJ, 2004).

[3] H. S. Carslaw and J. C. Jaeger, Conduction of heat in solids, 2nd edn. (Clarendon Press, Oxford, 1959).

[4] F. D. Gakhov, Boundary value problems (Pergamon Press, New York, 1966).

[5] J. A. Kolodziej and T. Strek, "Analytical approximations of the shape factors for conductive heat flow in circular and regular polygonal cross-sections", Intl J. Heat Mass Transfer 44 (2001) 999-1012; doi:10.1016/S0017-9310(00)00162-9.

[6] R.-L. Lin, "Explicit full field analytic solutions for two-dimensional heat conduction problems with finite dimensions", Intl J. Heat Mass Transfer 53 (2010) 1882-1892; doi:10.1016/j.ijheatmasstransfer.2009.12.070.

[7] W. Manners, "Heat conduction through irregularly spaced plane strip contacts", J. Mech. Engrg. Sci. Part C 214 (2000) 1049-1957; doi:10.1243/0954406001523515.

[8] J. C. Mason and D. C. Handscomb, Chebyshev polynomials (Chapman \& Hall/CRC, Boca Raton, FL, 2003).

[9] Yu. V. Obnosov, "A generalized Milne-Thomson theorem”, Appl. Math. Lett. 19 (2006) 581-586; doi:10.1016/j.ami.2005.08.006.

[10] Yu. V. Obnosov, "Three-phase eccentric annulus subjected to a potential field induced by arbitrary singularities", Quart. Appl. Math. 69 (2011) 771-786; doi:10.1090/S0033-569X-2011-01242-8.

[11] Yu. V. Obnosov, R. G. Kasimova, A. Al-Maktoumi and A. R. Kacimov, "Can heterogeneity of the near-wellbore rock cause extrema of the Darcian fluid inflow rate from the formation (the Polubarinova-Kochina problem revisited)? Comput. Geosci. 36 (2010) 1252-1260; doi:10.1016/j.cageo.2010.01.014.

[12] Yu. V. Obnosov, R. G. Kasimova and A. R. Kacimov, "A well in a 'target' stratum of a twolayered formation: the Muskat-Riesenkampf solution revisited", Transp. Porous Media 87 (2011) 437-457; doi:10.1007/s11242-010-9693-6.

[13] J. R. Philip, "Periodic nonlinear diffusion: an integral relation and its physical consequences", Aust. J. Phys. 26 (1973) 513-519.

[14] P. Ya. Polubarinova-Kochina, Theory of ground-water movement (Princeton University Press, Princeton, NJ, 1962).

[15] D. J. Sailor, D. Hutchinson and L. Bokovoy, "Thermal property measurements for ecoroof soils common in the western U.S.", Energy Build. 40 (2008) 1246-1251.

[16] S. Wolfram, Mathematica. A system for doing mathematics by computer (Addison-Wesley, Reading, MA, 1991). 